\title{
DEMARCAÇÃO DE UMA GRADE DE TRILHAS NO CENTRO DE PESQUISAS DA MATA DO PARAÍSO, VIÇOSA, MINAS GERAIS ${ }^{1}$
}

\begin{abstract}
Rômulo Ribon ${ }^{2}$
RESUMO - O Centro de Pesquisas da Mata do Paraíso (CPMP) é parte integrante do maior fragmento de Floresta Semidecidual Altimontana das imediações do município de Viçosa, sudeste de Minas Gerais. Como centro de pesquisas da Universidade Federal de Viçosa, ele tem servido para a coleta de dados de monografias de graduação, dissertações, teses e pesquisas de professores, principalmente dos Departamentos de Biologia Animal, Biologia Vegetal e Engenharia Florestal. Essas pesquisas têm sido conduzidas em trilhas preexistentes, aleatoriamente distribuídas pela mata, ou em novas trilhas abertas conforme a conveniência dos pesquisadores ou a exigência de suas pesquisas. Essa prática impede ou dificulta o mapeamento adequado de locais de amostragem e os monitoramentos temporal e espacial de comunidades animais e vegetais, bem como a repetibilidade de experimentos. No presente trabalho, descreve-se a implantação de um sistema de trilhas em 40 ha do CPMP, formado por um conjunto de cinco trilhas paralelas de $1.000 \mathrm{~m}$ e $11 \mathrm{de} 400 \mathrm{~m}$, formando 40 quadrados contíguos de 1 ha. O CPMP passou a ser uma das poucas reservas florestais no país a contar com um sistema ordenado e medido de trilhas, abrindo um novo leque de oportunidades para a realização de pesquisas de importância internacional. Destacam-se a urgência de manutenção das trilhas, a necessidade de seu georreferenciamento e a expansão da grade para 100 ha, a fim de que alcance padrões internacionais entre estações de pesquisa de comunidades florestais na região neotropical.
\end{abstract}

Palavras-chave: Ecologia, plots de longa duração, Mata Atlântica e Zona da Mata.

\section{PLOTTING OF A TRAIL GRID SYSTEM IN THE MATA DO PARAÍSO RESEARCH CENTER, VIÇOSA, STATE OF MINAS GERAIS}

\begin{abstract}
Mata do Paraíso Research Center (CPMP) is the core area of the largest Lower Montane Semideciduous Atlantic Forest fragment in Viçosa region, southeastern State of Minas Gerais, Brazil. As a research center belonging to the Universidade Federal de Viçosa (UFV), it has been an important site for the fieldwork of undergraduate monographs, Master and Doctorate Thesis, and research conducted by professors, mostly from the Animal Biology, Forestry and Plant Biology Departments. Those researches have been conducted along the existent trails randomly distributed in the forest or along new trails opened according to the research needs or the scientists' convenience. This management system imposes difficulties or even prevents the proper mapping of sampling locations, the temporal and spatial monitoring of animal and plant communities, and the repeatability of experiments. This paper describes a recently opened 40 ha trail grid system at the CPMP. The grid is composed by five parallel 1,000 m trails and eleven $400 \mathrm{~m}$ trails encompassing 40 squares of one hectare. The CPMP is now one of the few Brazilian forest research stations with a trail grid system, which allows scientists to conduct research in many different areas. This work highlights the urgency of trail maintenance and its proper, permanent marking, as well as its georeferencing and its expansion to encompass 100 hectares in order to meet international standard among other research stations in the Neotropics.
\end{abstract}

Key words: Atlantic Forest, ecology, long-term plots and Zona da Mata.

\footnotetext{
${ }^{1}$ Recebido em 20.02.2003 e aceito para publicação em 25.11.2004.

${ }^{2}$ Departamento de Ciências Biológicas da UFOP. E-mail: <ribon@iceb.ufop.br>.
} 


\section{INTRODUÇÃO}

As pesquisas sobre distribuições temporal e espacial de animais e plantas da região neotropical vêm aumentando continuamente. Entre inúmeros outros aspectos estudados, algumas pesquisas têm abordado a distribuição geográfica de uma única espécie (RIBON e MALDONADO-COELHO, 2001; REMSEN, 2001; RIBON et al., 2001; STOUFFER, 2001) ou grupo de espécies (COSTA e LEITE, 2000; OJEDA et al., 2000). Outros estudos têm enfocado a distribuição de comunidades de plantas (CLARK, 1998; CLARK et al., 1995, 1999) e animais, abordando questões comportamentais (ROBINSON e TERBORGH, 1995), de uso de habitat (GREENBERG e GRADWOHL, 1986) e de distribuições horizontal e vertical (TERBORGH et al., 1990; THIOLLAY, 1994; ROBINSON et al., 2000).

Essas pesquisas têm feito avançar consideravelmente o conhecimento que se tem de florestas tropicais, sobretudo no que diz respeito à ecologia de comunidades, ecologia de populações e comportamento animal. Apesar de abordarem diferentes aspectos, de terem sido realizadas com diferentes organismos e feitas em diferentes regiões, tais pesquisas têm pelo menos um ponto em comum: as coletas dos dados que as originaram foram proporcionadas por sistemas de trilhas devidamente ordenados e medidos, demarcados em mapas e bem marcados em campo, algumas vezes georreferenciados.

O Centro de Pesquisas da Mata do Paraíso (CPMP), sob o gerenciamento do Departamento de Engenharia Florestal da Universidade Federal de Viçosa (UFV), tem servido a vários departamentos da Instituição e de outras universidades e sido palco da coleta parcial ou total de dados para monografias de graduação (MELO, 1995; RESENDE, 1998; RIBON, 1994), dissertações (PAULA, 1996; ANCIÃES, 1998; RIBON, 1998; MALDONADO-COELHO, 2000; NASCIMENTO, 2002) e teses (MARANGON, 1999; RIBON, 2003) ao longo das últimas três décadas, bem como de coleta de espécimes em décadas anteriores (ERIKSON e MUMFORD, 1976). As pesquisas realizadas dependem, na maioria das vezes, do uso de trilhas. Embora utilizadas por vários pesquisadores (observação pessoal), aquelas atualmente existentes apresentam trajeto irregular, o que dificulta sua mensuração e o mapeamento dos pontos amostrais e das próprias trilhas. Essa falta de mensuração e a ausência de plotagem exata em um mapa também dificultam, ou impedem, tanto análises espaciais quanto temporais de distribuição dos organismos estudados. Por sua vez, essas limitações dificultam ou não permitem o aperfeiçoamento de pesquisas atuais e futuras, em especial aquelas visando aos monitoramentos espacial e temporal com amostras coletadas exatamente nos mesmos locais.

O presente trabalho objetivou contribuir para a melhoria da infra-estrutura de pesquisa do CPMP, descrevendo e disponibilizando para a comunidade científica um sistema ordenado de trilhas recentemente implantado na área. O trabalho também visou destacar a importância desse sistema de trilhas e de sua manutenção e marcação adequadas, permitindo que várias pesquisas realizadas no local sejam otimizadas temporal e espacialmente.

\section{2. ÁREA DE ESTUDO}

O CPMP, com 194 ha, situa-se entre as coordenadas $20^{\circ} 46^{\prime}$ e $20^{\circ} 50^{\prime} \mathrm{S}$ e $42^{\circ} 51^{\prime}$ e $42^{\circ} 49^{\prime} \mathrm{O}$ e é a porção mais bem preservada de um fragmento florestal de 384 ha orientado na direção sudeste-noroeste, a sudeste do município de Viçosa, Minas Gerais. A área constitui o maior fragmento florestal da região (PEREIRA et al., 2001; RIBON, 1998), com altitudes variando entre 690 e $870 \mathrm{~m}$. Conhecida antigamente como Fazenda Paraíso e Córrego São Benedito, sofreu desmatamentos no passado por parte de seus antigos proprietários, com vistas à plantação de café. Após passar para a responsabilidade da Prefeitura Municipal de Viçosa, sofreu ainda intensa exploração madeireira, caça, captura de aves e invasão de gado (Sr. Raimundo Stanciola de Abreu, antigo zelador da área-comunicação pessoal). Há 37 anos, ao passar para a responsabilidade da UFV (INFORMATIVOUREMG, 1966), cessou a retirada de madeira e diminuíram as caçadas, mas as invasões de gado na área de capim-gordura, embora diminuíssem, continuaram até cerca de sete anos atrás, quando também foi feito um aceiro com trator, delimitando-se o perímetro da área pertencente à UFV. Ainda assim, um incêndio atingiu pequena parte da área em 1999.

A vegetação predominante do CPMP é de mata secundária, nos estados inicial, médio e avançado de regeneração (BRASIL, 1993), do tipo Floresta Semidecidual Altimontana(OLIVEIRA-FILHOe FONTES, 2000), 
também denominada Floresta Estacional Semidecidual Montana (VELOSO et al., 1991) ou, ainda, Mata Atlântica de Montanha (STOTZ et al., 1996). Apresenta também pequenas porções de brejo com taboa (Typha $\mathrm{sp.),}$ pastagem abandonada de capim-gordura (Melinis minutiflora) e um pequeno açude. A área comporta hoje a maior extensão contínua de floresta nativa em baixadas e grotas de toda a região (RIBON, 1998), havendo também vegetação florestal nas encostas e topos de morro.

\section{MATERIAL E MÉTODOS}

A grade de trilhas foi delimitada com vistas ao estudo da territorialidade de aves de sub-bosque (RIBON, 2003), abrangendo 40 ha do extremo sudeste-sudoeste da mata (Figura 1). O perímetro da área foi inicialmente traçado na ortofotocarta $n^{\circ} 43-21-07$, produzida pela Companhia Energética de Minas Gerais (CEMIG) em 1987, passando-se, em seguida, para a abertura e mapeamento da grade de trilhas em campo.

O ponto inicial para a grade, a $690 \mathrm{~m}$ de altitude (medição com altímetro Sunoh SAL7030), foi estabelecido na estrada após a clareira onde atualmente se constrói um centro de visitantes e de apoio à pesquisa, cerca de $50 \mathrm{~m}$ a sudeste deste. As trilhas foram abertas por dois auxiliares de campo e medidas, juntamente com as curvas de nível, por um topógrafo e um auxiliar entre 29 de maio e 4 de julho de 2000 , em 34 dias/homem de trabalho. Os dados topográficos foram mapeados em computador, com curvas de nível demarcadas a cada $10 \mathrm{~m}$, a um custo aproximado de US\$ 0.30 (trinta centavos de dólar) por metro linear de trilha aberta.

Cada trilha recebeu uma marcação temporária em intervalos irregulares de distância e marcações em cada um dos cruzamentos com as demais trilhas, de modo que os vértices de quadrados de 100 x 100 m foram marcados com sacos plásticos atados a estacas pequenas. As marcações foram feitas com fitas plásticas de cores fortes grafadas com caneta de retroprojetor. De acordo com sua extensão e direção, cada trilha recebeu uma letra ou um número como denominação. Trilhas maiores, orientadas no sentido sudeste-noroeste, foram definidas por letras, e trilhas menores, no sentido sudoeste-nordeste, foram definidas por números.

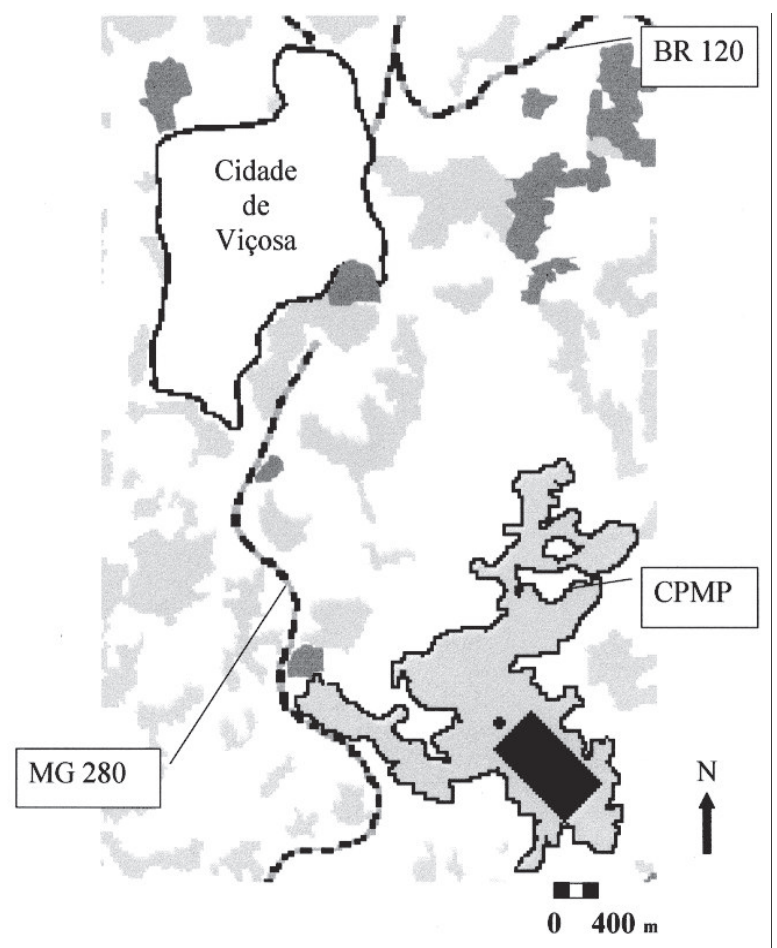

Figura 1 - Localização do Centro de Pesquisas da Mata do Paraíso (CPMP) na região de Viçosa, Minas Gerais. O retângulo preto indica a localização aproximada da grade de trilhas de 40 ha. O ponto a noroeste do retângulo indica o Centro de Visitantes. Em cinza, outros fragmentos de floresta nativa; em preto, plantios de eucalipto.

Figure 1 - Location of the Mata do Paraíso Research Center (CPMP), at Viçosa region, Minas Gerais state. The rectangle indicates the approximate location of the 40ha grid. The dot at Northwest from the rectangle indicates the Visitors Center. Gray patches are other native forest fragments and dark gray patches, eucalyptus plantations.

\section{RESULTADOS E DISCUSSÃO}

\subsection{Resultados}

A grade de trilhas abrange um perímetro de 2.800 $\mathrm{m}$ compreendendo cinco trilhas de $1.000 \mathrm{~m}$, no sentido sudeste-noroeste (trilhas A, B, C, D e E) e 11 trilhas de $400 \mathrm{~m}$ sudoeste-nordeste (trilhas de números 1 a 11), que se cruzam a cada $100 \mathrm{~m}$, formando 40 quadrados de 1 ha. No total, $9.400 \mathrm{~m}$ de trilhas ficaram disponíveis para uso. Aquela iniciada no meio da antiga estrada, onde a altitude foi medida, recebeu a

R. Árvore, Viçosa-MG, v.29, n.1, p.151-158, 2005 
denominação $\mathrm{C}$, ficando as trilhas $\mathrm{A}$ e $\mathrm{B}$ à direita de quem avança pela estrada, vindo do centro de visitantes (a noroeste), e as trilhas D e E à esquerda. Ligando os pontos iniciais das trilhas A a E, tem-se a trilha 1 e, daí em diante, sempre paralela a esta e a cada 100 $\mathrm{m}$, as outras 10 trilhas de $400 \mathrm{~m}$ (Figura 2).

Os quadrantes delimitados por cada quatro segmentos de trilhas foram numerados, tomando-se como referência a trilha de $1.000 \mathrm{~m}$ mais à direita (a sudoeste) e a trilha de $400 \mathrm{~m}$ mais próxima do centro de visitantes (a noroeste). Dessa forma, o quadrante no extremo noroeste da grade de trilhas recebeu a denominação DE1 (Figura 3). Cada segmento de $100 \mathrm{~m}$ de trilha recebeu denominação e marcação semelhantes no campo. Desse modo, os primeiros $100 \mathrm{~m}$ da trilha C foram marcados com fitas com a inscrição "C1", enquanto o segmento de 0 a $100 \mathrm{~m}$ da trilha $\mathrm{A}$, indo para a trilha $\mathrm{B}$, recebeu fitas com o escrito $\mathrm{AB} 1$, de 100 a 200 m, AB2 de 200 a 300 m, e assim sucessivamente.

A grade de trilhas abrangeu trechos de relevo suave, como toda a baixada da porção sudeste da Mata do Paraíso (quadrantes BC8, CD8, BC9, CD9), e trechos de relevo extremamente íngreme (quadrantes CD2 a CD6) (Figura 2). A maior parte da grade foi plotada sobre floresta em estádio avançado de regeneração, mas houve cerca de 1 ha dominado por Pinus sp. (parte dos quadrantes BC1 e BC2), 0,5 ha com árvores esparsas de Eucalyptus sp. (quadrante CD4) (ambos com subbosque em desenvolvimento), 1,0 ha de samambaias (Pteridium aquilinun) (quadrantes AB 1 e AB2; DE1) e cerca de 6 ha com sub-bosque dominado por taquarais nativos (quadrantes CD3, CD4, DE3, DE4, AB7, AB9 e AB10) (uma touceira de bambu exótico, no entanto, encontra-se no quadrante CD2).

Após duas limpezas em intervalos de seis meses, as trilhas não receberam mais nenhum cuidado ou remarcação, estando desde maio de 2002 sem manutenção e se fechando, em virtude da regeneração da vegetação e perda das marcações temporárias. A fim de incentivar o uso do sistema de trilhas por pesquisadores, a posição da grade e o detalhamento das trilhas e curvas de nível são mostrados neste estudo. A fim de facilitar o trabalho de outros pesquisadores, o arquivo digital para uso em computador encontra-se atualmente nas secretarias dos Departamentos de Biologia Animal, Biologia Vegetal e Engenharia Florestal da UFV.

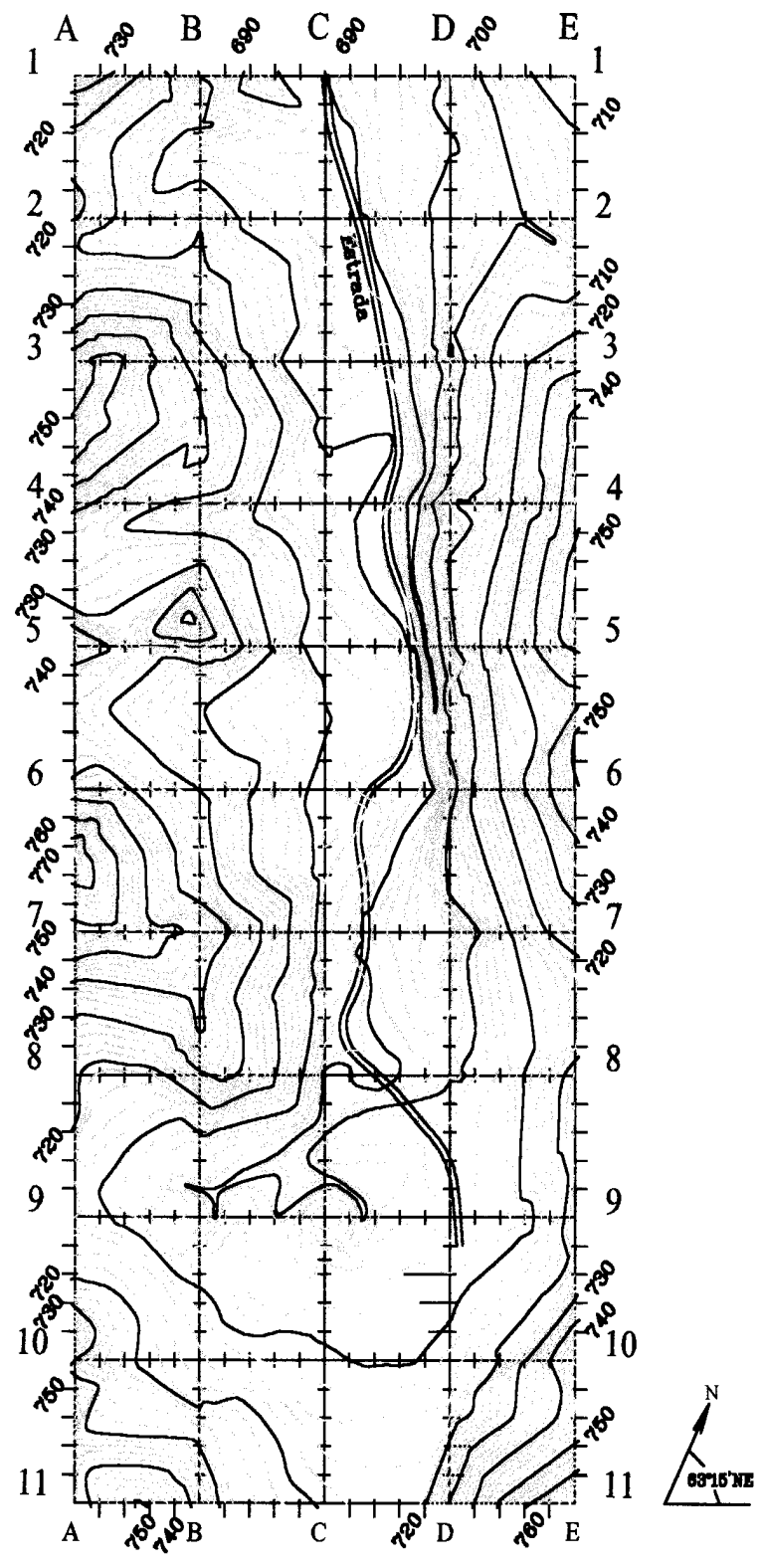

Figura 2-Grade de trilhas do Centro de Pesquisas da Mata do Paraíso. Letras e números nas extremidades de linhas retas indicam as trilhas de $1.000 \mathrm{~m}$ e $400 \mathrm{~m}$, respectivamente. Centenas na parte exterior da grade indicam algumas curvas de nível (altitude aproximada.

Figure 2-Gird trail system at the Mata do Paraíso Research Center. Letters and numbers at the end of straight lines are 1.000 and 400 meters trails respectively. Skewed numbers outside the grid are the values of contour lines. 


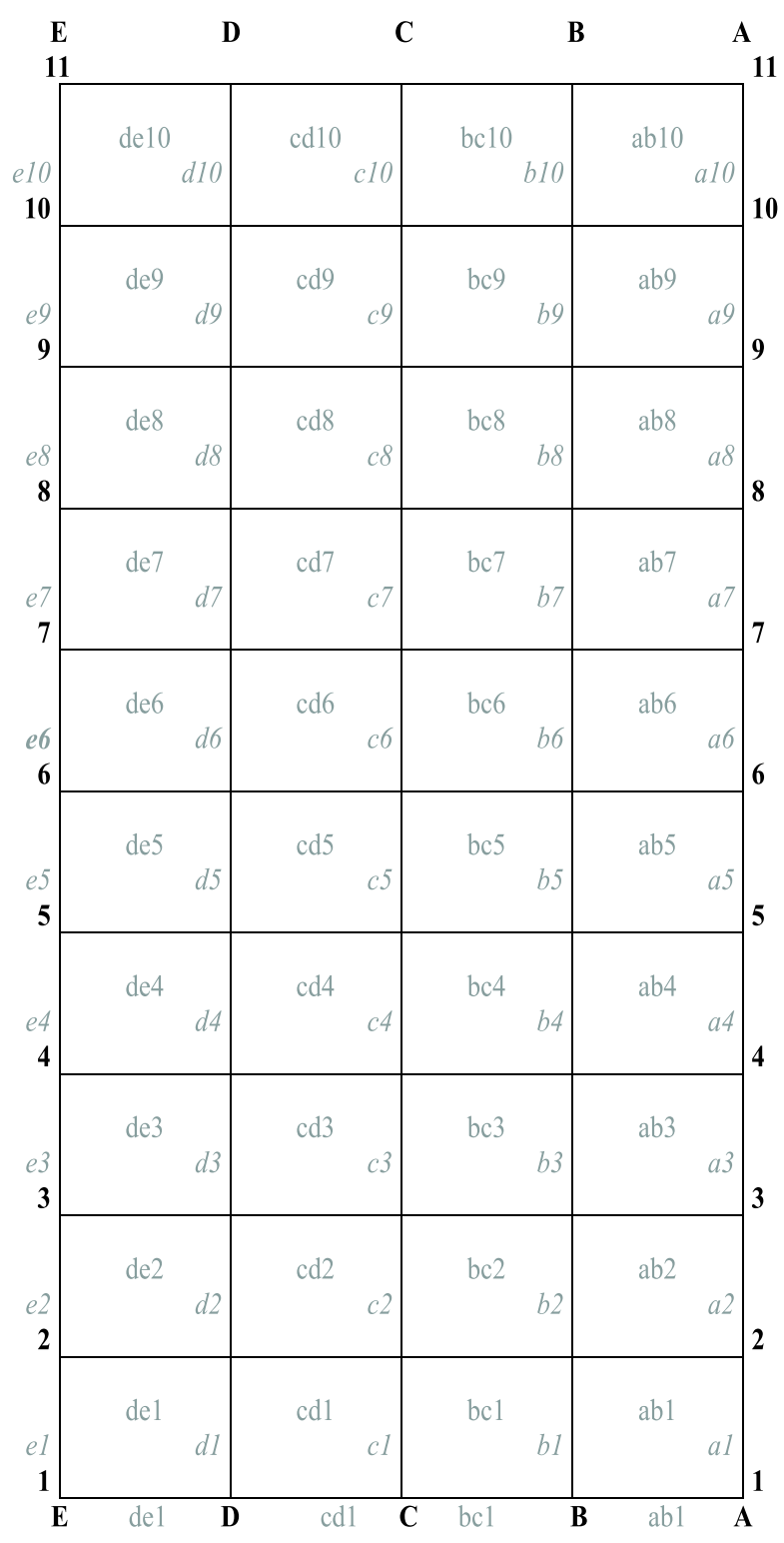

Figura 3 - Sistema de marcação da grade de trilhas do Centro de Pesquisas da Mata do Paraíso. Letras e números em negrito: código de cada trilha de $1.000 \mathrm{~m} \mathrm{e}$ 400 m, respectivamente; códigos em cinza indicam os quadrantes de 1 ha; códigos em cinza e itálico: segmentos de $100 \mathrm{~m}$ de cada trilha.

Figure 3 -Marking of the trail system at Mata do Paraíso Research Center. Letters and numbers in bold indicate the codes of each $1,000 \mathrm{~m}$ and $400 \mathrm{~m}$ trails, respectively; gray codes indicate one-hectare squares; gray and italic codes are $100 \mathrm{~m}$ segments of each trail.

\subsection{Discussão}

A implementação da grade de trilhas da Mata do Paraíso é importante para sua otimização enquanto estação de desenvolvimento de pesquisas científicas em áreas como Zoologia, Ecologia (de populações, comunidades e ecossistemas), Ecologia Comportamental, Ecofisiologia, Pedologia e Climatologia, entre outras. A utilização do sistema de trilhas aqui apresentado permitirá aos pesquisadores a plotagem exata de seus locais de coleta, facilitando sua localização durante a execução de suas investigações. Talvez ainda mais importante, será possível para outros pesquisadores, no futuro, coletar dados nos exatos locais onde pesquisadores contemporâneos venham a trabalhar, permitindo comparações temporais e espaciais.

O sistema de trilhas aqui descrito coloca a Mata do Paraíso como a única unidade de conservação no Estado de Minas Gerais e uma das poucas do país a ter um sistema de referenciamento espacial detalhado em área de Mata Atlântica para servir à pesquisa científica. Essa idiossincrasia se deve, em grande parte, aos custos envolvidos e à dificuldade em se estabelecer um sistema de trilhas em área de mata secundária e com relevo acidentado. Adicionalmente, a histórica utilização da Mata do Paraíso como centro de pesquisas sobre diversos aspectos da Mata Atlântica e seu acesso facilitado também foram determinantes para que fosse escolhida para o estabelecimento do sistema de trilhas.

Os trabalhos de Clark (1998) e Clark et al. (1995, 1999) sobre distribuição espacial de palmeiras e dicotiledôneas na Estação Biológica de La Selva, na Costa Rica, recentemente revelaram distintos padrões de distribuição de plantas em diferentes porções do relevo. Os dados, coletados em uma grade de trilhas georreferenciada de 500 ha, incrementaram o rumo de pesquisas na área de solos e botânica em florestas tropicais e deram novo impulso ao estudo das interações entre microtopografia e botânica. Na Estação Biológica de Cocha Cashu, no Parque Nacional de Manu, Peru, Terborgh et al. (1990), através do mapeamento de territórios e aplicação de outras técnicas de levantamento quantitativo de aves, em um sistema de trilhas de 97 ha, deram a primeira caracterização completa de uma comunidade de aves em uma floresta neotropical. Eles forneceram também parte dos poucos dados sobre o tamanho dos territórios de aves 
neotropicais (245 espécies). Utilizando a mesma infra-estrutura de trilhas, Robinson e Terborgh (1995) obtiveram dados sobre aspectos comportamentais importantes para o entendimento da agressão interespecífica de aves florestais, aumentando a compreensão da ciência sobre os fatores que permitem a coexistência de um número tão grande de aves na Amazônia. Em uma grade de trilhas de 100 ha na Estação de Campo de Nouragues, sul da Guiana Francesa, Thiollay (1994) fez a segunda descrição completa de uma comunidade de aves da Amazônia, sendo seguido no mesmo tipo de pesquisa por Robinson et al. (2000), que utilizaram o sistema de trilhas da ilha de Barro Colorado, no Panamá. Utilizando as trilhas dessa illha, Greenbergh e Gradwohl (1986) foram dos primeiros a descrever o território de aves neotropicais e sua permanência nos mesmos territórios no longo prazo.

Em florestas tropicais, a distribuição em manchas e a raridade de espécies é marcante (TERBORGH et al., 1990; CLARK et al., 1999). Em vista disso, Thiollay (1994) e Robinson et al. (2000) advertiram para o fato de que amostragens em sistemas de grades muito pequenos podem afetar fortemente a percepção e descrição de comunidades. Desse modo, acompanhando a tendência dos trabalhos de Terborgh et al. (1990), eles orientaram para que pesquisas sobre comunidades de florestas tropicais fossem feitas em sistemas de trilhas nunca menores do que 100 ha. A questão da escala para o entendimento de padrões e processos em ecologia é também enfatizada por Wiens (1976, 1994) e Turner (1989).

Essa discussão dá uma idéia do grande potencial que o sistema de trilhas do CPMP possui para o desenvolvimento de pesquisas de importância global nos diversos ramos da ecologia e várias outras áreas da ciência. No entanto, é urgente que a administração do CPMP providencie a limpeza das trilhas e marcações definitivas e de fácil visualização em seus pontos de cruzamento, bem como marcações permanentes menores, preferencialmente a cada $10 \mathrm{~m}$. Ações adicionais a serem tomadas consistem no mapeamento detalhado da vegetação de cada um dos quadrantes (preferencialmente com enfoque fitossociológico) e no georreferenciamento de cada um dos 55 pontos de cruzamento de trilhas.

A necessidade de estudos de longo prazo foi recentemente reconhecida por Holmes e Sherry (2001). Tal visão é compartilhada por outros grupos de pesquisa que estão iniciando a implementação de estações de monitoramento biológico no longo prazo em várias regiões do mundo [Tropical Ecology, Assessment, and Monitoring Initiative (Team) da Organização NãoGovernamental Conservation International e workshop "Empowering basic and applied conservation research and action from large-scale, long-term plots in the Neotropics", realizado durante a III Conferência Ornitológica Norte-Americana, em New Orleans, EUA, em setembro de 2002].

Seguindo essa tendência e acatando as recomendações de Thiollay (1994) e Robinson et al. (2000), o Departamento de Engenharia Florestal, como responsável pela Mata do Paraíso, deveria melhorar o sistema de trilhas aqui descrito e fazer todo o esforço para sua expansão, a fim de que abranja pelo menos 100 ha. Sua manutenção, marcação adequada e expansão para 100 ha deveriam ser tomadas como condição de infra-estrutura sine qua non para que a Mata do Paraíso se coloque definitivamente como uma estação de pesquisas de destaques nacional e internacional.

Em uma época em que dotações orçamentárias à pesquisa vão sendo cada vez mais condicionadas a publicações em periódicos científicos de alto impacto, as referidas sugestões deveriam ser levadas em conta sem muito protelamento.

\section{AGRADECIMENTOS}

$\mathrm{O}$ autor agradece as seguintes instituições e pessoas: Programa Natureza e Sociedade / Fundo Mundial para a Natureza - Universidade Estadual de Nova Iorque (WWF/SUNY) / "Projeto Tovacuçu: história natural e distribuição de aves em fragmentos de Mata Atlântica de encosta do Brasil" (WWF CSR 142-00); Antônio Carlos Ottoni, que se empenhou na abertura e medição das trilhas e sua plotagem no computador; Sinval Lopes Ribeiro (in memoriam) e Fernando Stanciola, que auxiliaram a abertura e manutenção das trilhas; Emílio C. A. Neto e Natália R. Neves, que colaboraram na marcação das trilhas; e três revisores anônimos que contribuíram para a melhoria do artigo. Esta é a publicação no 5 do Projeto "Ecologia e Biogeografia da Avifauna Silvestre de Minas Gerais" (UFV $\left.n^{\circ} 20.401 .649 .754\right)$ e produto da tese de doutorado de RR. O Programa de Pós-Graduação em Ecologia, Conservação e Manejo de Vida Silvestre é apoiado pelo U.S. Fish and Wildlife Service e contribui para a implementação da convenção para a proteção da flora 
e fauna e das belezas cênicas dos países das Américas (1940) e da Convenção sobre Áreas Úmidas (RAMSAR, Irã, 1971) na América Latina e no Caribe.

\section{REFERÊNCIAS BIBLIOGRÁFICAS}

ANCIÃES, M. Assimetria flutuante como indicador de efeitos da fragmentação em Passeriformes da Mata Atlântica. 1998. 98 f. Dissertação (Mestrado em Ecologia, Conservação e Manejo de Vida Silvestre) Universidade Federal de Minas Gerais, Belo Horizonte, 1998.

CLARK, D. A. Deciphering landscape mosaics of neotropical tress: GIS and systematic sampling provide new views of tropical rainforest diversity. Annals of the Missouri Botanical Garden, v. 85, p.18-33, 1998.

CLARK, D. A.; CLARK, D. B.; SANDOVAL, R. M. Edaphic and human effects on landscape-scale distributions of tropical rain forest palms. Ecology, v. 76, n. 8, p. 2581-2594, 1995.

CLARCK, D. B., PALMER, M. W.; CLARCK, D. A. Edaphic factors and the landscape-scale distribution of tropical rain forest trees. Ecology, v. 80, n.8, p.2666-2675, 1999.

COSTA, L. P.; LEITE, Y. L. R. Biogeography of South American Forest mammals: endemism and diversity in the Atlantic Forest. Biotropica, v. 32 , n. 4b, p. 872-881, 2000.

BRASIL. Resolução $\mathbf{n}^{\circ}$. 10 de $\mathbf{1}^{\circ}$ de outubro de 1993. Diário Oficial da República Federativa do Brasil. Conselho Nacional do Meio Ambiente. Brasília. 03/11/1993. p. 16.497-16.498, 1993.

ERIKSON, H. T.; MUMFORD, R. E. Notes on birds of Viçosa, Brazil Region. Station Bulletin. West Lafayette; Purdue University, v. 131, 29 p. 1976.

GREENBERG, R.; GRADWOHL, J. Constant density and stable territoriality in some tropical insectivorous birds. Oecologia, v. 69, p. 618$625,1986$.

HOLMES, R. T.; SHERRY, T. W. Thirty-year bird population trends in an unfragmented temperate deciduous forest: importance of habitat change. The Auk, v. 118, n. 3, p. 589-609, 2001.
ASSINADO o primeiro convênio entre a UREMG e a Prefeitura Municipal de Viçosa. Informativo UREMG, n.16, p. 1, 1966.

MALDONADO-COELHO, M. Efeitos da fragmentação florestal em bandos mistos de aves de Mata Atlântica, no sudeste de Minas Gerais. 2000. 120 f. Dissertação (Mestrado em Ecologia, Conservação e Manejo de Vida Silvestre) - Universidade Federal de Minas Gerais, Belo Horizonte, 2000.

MARANGON, L. C. Florística e fitossociologia de área de floresta semidecidual visando dinâmica de espécies florestais arbóreas no município de Viçosa, MG. 1999. 147 f. Tese (Doutorado em Ecologia e Recursos Naturais) - Universidade Estadual Paulista, São Carlos, 1999.

MELO, F. R. Densidade, tamanho e composição de grupos de Callicebus personatus (Cebidae: primates) em fragmentos florestais do município de Viçosa, Minas Gerais. 1995. 29 f.

Monografia (Estágio Supervisionado em Biologia BIO 498) - Universidade Federal de Viçosa, Viçosa, 1995.

NASCIMENTO, I. C. Fenologia do vôo nupcial e amostragem de comunidade de formigas (Hymenoptera: Formicidae) em área de Mata Atlântica do município de Viçosa Minas Gerais. 2002. 76 f. Dissertação (Mestrado em Entomologia) - Universidade Federal de Viçosa, Viçosa, 2002.

OJEDA, R. A.; BLEDINGER, P. G.; BRANDL, R. Mammals in South American drylands: similarity and trophic structure. Global Ecology \& Biogeography, v. 9, p.115-119, 2000.

OLIVEIRA-FILHO, A. T.; FONTES, M. A. Patterns of floristic differentiation among Atlantic Forests in southeastern Brazil and influence of climate. Biotropica, v. 32, n. 4b, p. 793-810, 2000.

PAULA, A. L. Biodiversidade e análise faunística de Heteroptera da Mata do Córrego do Paraíso, Viçosa, Minas Gerais. 1996. 95 f. Mestrado (Entomologia) Universidade Federal de Viçosa, Viçosa, 1996. 
PEREIRA, R. A., et al. Caracterização da paisagem, com ênfase em fragmentos florestais, no município de Viçosa, Minas Gerais. Revista Árvore, v. 25, n. 3, p. 327-333, 2001.

REMSEN Jr., J. V. True winter range of the Veery (Catharus fucescens): lessons for determining winter ranges of species that winter in the tropics. The Auk, v. 118, n. 4, p. 838-848, 2001.

RESENDE, D. C. Comportamento territorial e seleção de locais para defesa de território em Libelulidae (Odonata). 1994. 31 f. Monografia (Estágio Supervisionado em Biologia - BIO 498) Universidade Federal de Viçosa, Viçosa, 1998.

RIBON, R. Comportamento do estalador (Corythopis delalandi LESSON, 1830), (PASSERIFORMES: TYRANNIDAE) em Viçosa, Minas Gerais: território e relações intra- e inter-específicas. 1994. $44 \mathrm{f}$. Monografia (Estágio Supervisionado em Biologia - BIO 498) - Universidade Federal de Viçosa, Viçosa, 1994.

RIBON, R.; MALDONADO-COELHO, M. A range extension for Slender Antbird Rhopornis ardesiaca (Wied, 1831) with some comments on external morphology of adults. Cotinga, v. 16, p. 52-56, 2001.

RIBON, R. Fatores que influenciam a distribuição da avifauna em fragmentos de Mata Atlântica nas montanhas de Minas Gerais. 1998. $127 \mathrm{f}$. Dissertação (Mestrado em Ecologia, Conservação e Manejo de Vida Silvestre) - Universidade Federal de Minas Gerais, Viçosa, 1998.

RIBON, R. Aves em fragmentos de Mata Atlântica do sudeste de Minas Gerais: incidência, abundância e associação à topografia. 2003. $127 \mathrm{f}$. Tese (Doutorado em Ecologia, Conservação e Manejo de Vida Silvestre) - Universidade Federal de Minas Gerais, Belo Horizonte, 2003.

RIBON, R.; WHITNEY, B. M.; PACHECO, J. F. The discovery of the Bahia Spinetail Synallaxis cinerea in northeastern Minas Gerais, Brazil, with additional records of some rare and threatened montane Atlantic Forest birds. Cotinga, v.17, p. 46-50, 2001.

R. Árvore, Viçosa-MG, v.29, n.1, p.151-158, 2005
ROBINSON, S. K.; TERBORGH, J. Interspecific aggression and habitat selection by Amazonian birds. Journal of Animal Ecology, v. 64, p. 1-11, 1995.

ROBINSON, W. D.; BRAWN, J. D.; ROBINSON, S. $\mathrm{K}$. Forest bird community structure in central Panama: influence of spatial scale and biogeography. Ecological Monographs, n. 70, p. 209-235, 2000.

STOTZ, D. F. et al. Neotropical birds: ecology and conservation. Chicago: University of Chicago Press, 1996. 480 p.

STOUFFER, P. C. Do we know what we think we know about winter ranges of migrants to South America? the case of the Veery (Catharus fuscescens). The Auk, v. 118, n. 4, p. 832-837, 2001.

TERBORGH, J. et al. Structure and organization of an Amazonian forest bird community.

Ecological Monographs, v.60, n.2, p.213238, 1990.

THIOLLAY, J.-M. Structure, density, and rarity in an Amazonian rainforest bird community.

Journal of Tropical Ecology, n.10, p. 449$481,1994$.

TURNER, M. G. Landscape ecology: the effect of pattern on process. Annual Review of Ecology and Systematics, v.20, p. 171-197, 1989.

VELOSO, H. P.; RANGEL FILHO, A. L. R.; LIMA, J. C. A. Classificação da vegetação brasileira, adaptada a um sistema universal. Rio de Janeiro: IBGE - Instituto Brasileiro de Geografia e Estatística. Departamento de Recursos Naturais e Estudos Ambientais. Rio de Janeiro. 1991. 124 p.

WIENS, J. A . Population responses to patchy environments. Annual Review of Ecology and Systematics, v. 7, p. 81-120, 1976.

WIENS, J. A. Habitat fragmentation: island $v$ landscape perspectives on bird conservation. Ibis, v. 137S, p. 97-104, 1994. 